\title{
Effects of deep brain stimulation in the subthalamic nucleus or globus pallidus internus on step initiation in Parkinson disease
}

\author{
Laboratory investigation
}

\author{
Laura Rocchi, Ph.D., ${ }^{1}$ Patricia Carlson-Kuhta, Ph.D., ${ }^{2}$ Lorenzo Chiari, Ph.D., ${ }^{1}$ \\ Kim J. Burchiel, M.D., ${ }^{3}$ Penelope Hogarth, M.D., and Fay B. Horak, Ph.D. ${ }^{2}$ \\ ${ }^{1}$ Department of Electronics, Computer Science \& Systems, University of Bologna, Italy; and Departments of \\ ${ }^{2}$ Neurology and ${ }^{3}$ Neurological Surgery, Oregon Health \& Science University, Portland, Oregon
}

\begin{abstract}
Object. Difficulty with step initiation, called "start hesitation," is related to gait bradykinesia and is an early hallmark of gait freezing in Parkinson disease (PD). Authors of this study investigated the effects of deep brain stimulation (DBS) and levodopa on step initiation before and 6 months after DBS surgery in 29 patients with PD who were randomized to either the bilateral subthalamic nucleus (STN) or globus pallidus internus (GPi) as the DBS site.

Methods. The authors measured the amplitude and duration of anticipatory postural adjustments (APAs), the feed-forward postural preparation that precedes the onset of voluntary step initiation, based on center-of-pressure displacements on a force plate. They also measured the length and velocity of the first step using a motion analysis system to study kinematics. Some of the patients (22) were from a large, multicenter, double-blind clinical trial, and all patients in the study (29, PD-DBS group) were randomized to DBS in either the bilateral STN (15 patients) or bilateral GPi (14 patients). Differences in step initiation were investigated in 2 conditions before surgery (off/on levodopa) and in 4 conditions after surgery (off/on levodopa combined with off/on DBS). Twenty-eight elderly healthy control volunteers (CTRL group) were also tested, and 9 control volunteers with PD who met the criteria for DBS (PD-C group) were tested at baseline and 6 months later.

Results. Patients in the PD-DBS group had smaller amplitudes and longer durations of APAs compared with those in the 28 healthy control volunteers in all conditions. Before surgery, APAs improved with levodopa. After surgery, the APAs were significantly worse than in the best-treatment state before surgery (DOPA condition), and responsiveness to levodopa decreased. No differences in APAs were detected between the STN and GPi groups. A comparison with PD control volunteers who did not undergo DBS surgery confirmed that a deterioration in step preparation was not related to disease progression.

Step length and velocity were smaller in the PD-DBS group than in controls in all conditions. Before surgery, levodopa improved the length and velocity of the first step. Both step length and velocity were unchanged in the best-treatment state before surgery (DOPA condition) as compared with after surgery (DBS+DOPA), with only step velocity in the STN group getting worse after surgery.

Conclusions. Six months of DBS in the STN or GPi impaired anticipatory postural preparation for step initiation, the opposite effect as with levodopa. Deep brain stimulation disrupted postural preparation more than step execution, suggesting independent motor pathways for preparation and execution of gait. Although turning the stimulators on after surgery combined with levodopa benefited the postural preparation to step, a comparison of pre- and postsurgery conditions suggests that either the surgery itself or 6 months of continuous stimulation may lead to an alteration of circuits or plastic changes that impair step initiation.

(http://thejns.org/doi/abs/10.3171/2012.8.JNS112006)
\end{abstract}

\section{Key Words - Parkinson disease anticipatory postural adjustment • functional neurosurgery}

$\mathrm{S}$ TEP initiation is impaired in people with PD, resulting in the clinically observed impairments of start hesitation and gait freezing. ${ }^{5,9}$ Studies have shown that problems with step initiation in PD are often caused by a reduced size of postural preparation for single limb support. ${ }^{19,36}$

Abbreviations used in this paper: APA = anticipatory postural adjustment; $\mathrm{COP}=$ center of pressure; DBS = deep brain stimulation; GPi = globus pallidus internus; PD = Parkinson disease; STN = subthalamic nucleus; UPDRS = Unified Parkinson's Disease Rating Scale.

\section{- deep brain stimulation \\ posture control - step initiation}

The feed-forward postural preparation that precedes the onset of voluntary movements, such as voluntary step initiation, is referred to as "anticipatory postural adjustments." ${ }^{5,6,10,26,31}$ Preparation for a voluntary step involves displacement of the COP (that is, application point of resultant ground reaction force) backward and laterally toward the swing limb that results in movement of the body center of mass forward and over the stance limb. ${ }^{5,31}$ Anticipatory postural adjustments are thought to involve separate neural circuitry from voluntary movements, with coupling between the postural preparation and voluntary step occurring in the medullary reticular formation 
that receives projections from the basal ganglia and is affected by PD. ${ }^{40,41}$ In addition, APAs probably involve the supplementary motor area, which is suppressed in patients with PD. ${ }^{18,22,31}$ The small APA amplitudes in patients with PD have been shown to significantly increase with levodopa, ${ }^{36}$ but the effects of DBS on step initiation is unclear. ${ }^{11,28}$

In the present study, we characterized both APAs prior to self-initiated steps and the steps themselves in 29 patients with PD before functional neurosurgery and 6 months after electrodes were implanted. As part of a Veterans Affairs/National Institute of Neurological Disorders and Stroke multicenter, double-blind clinical trial comparing target sites for DBS, patients were randomized to DBS surgery in either the STN or the GPi. ${ }^{44}$

Deep brain stimulation provides remarkable benefits and generally betters the same symptoms improved by levodopa in PD. ${ }^{2,25,38}$ Stimulation of the STN or GPi reduces bradykinesia, rigidity, tremor, and some gait difficulties. ${ }^{34}$ However, we recently found that DBS surgery impairs automatic postural responses to external perturbations, which are also not improved with levodopa. ${ }^{42}$ Impairment of automatic postural responses after DBS surgery is consistent with the observation that the number of falls may increase after DBS surgery, especially after electrodes are implanted in the STN ${ }^{16}$ Moreover, our recent meta-analysis showed that the postural instability and gait disorder subscore of the motor UPDRS continues to worsen 5 years after DBS surgery, especially in the STN, despite minimal long-term deterioration of the cardinal signs measured by the UPDRS..$^{43}$

In the present study, we investigated whether DBS has the same effect as levodopa in improving step initiation in PD. ${ }^{5,36}$ We evaluated step initiation in the levodopa on and off conditions both before surgery and 6 months after surgery when the short-term effects of surgery had stabilized. ${ }^{8}$ The aims of this study were to determine the effects of DBS on step initiation on and off levodopa and to compare the effects of DBS in the STN and GPi on step initiation.

\section{Methods}

\section{Patients and Controls}

Twenty-nine patients with idiopathic PD who underwent DBS surgery participated in this study (PD-DBS group, mean age $61.3 \pm 7.7$ years); 22 of these patients constituted a smaller cohort from a large, multicenter clinical trial of $\mathrm{DBS}^{44}$ and were recruited from the Seattle and Portland sites. All of the patients were randomized to 1 of 2 DBS surgical site groups: the GPi (14 patients) or the STN (15 patients).

There were 2 control groups: 28 healthy control volunteers (CTRL group, mean age 62.4 \pm 7.4 years) and 9 controls with PD (disease duration $11.6 \pm 6.3$ years) who met the criteria for, but did not undergo, DBS surgery (PD-C group, mean age $60.3 \pm 7.8$ years).

All participants in the study gave informed consent in accordance with the Oregon Health \& Science University and the Veterans Administration Medical Center Institutional Review Board regulations for human subject studies. Table 1 summarizes the clinical characteristics of patients in the PD-DBS group when entering the study.

\section{Surgical Procedure}

Patients in the PD-DBS group were randomly assigned (following a double-blind method) into either the STN (15 patients) or the GPi (14 patients) group. Both the patients and the experimenters remained unaware of the stimulation site for the duration of the multicenter study. The surgical procedure was performed by a neurosurgeon (K.J.B.) with extensive experience in DBS surgeries. Patients underwent surgical implantation of the DBS electrodes (model 3387, Medtronic, Inc.), which were inserted bilaterally through 2 precoronal sulcus bur holes. Intraoperative microelectrode recordings were performed to confirm target localization, and corrections to the implant site were made accordingly. One week after electrode implantation, a single- (Soletra, Medtronic, Inc.) or dualchannel (Kinetra, Medtronic, Inc.) internal pulse generator was surgically implanted in the infraclavicular area. Magnetic resonance imaging studies were available for 19 of the patients. For the STN, the target contact was the second contact (Contact 1), and for the GPI, the target contact was the most distal one (Contact 0$)$. The mean ( \pm standard deviation) distance of the target contacts relative to the anterior commissure-posterior commissure midpoint was as follows: $\mathrm{x}=11.24 \pm 1.45 \mathrm{~mm}, \mathrm{y}=-4.01 \pm$ $1.09 \mathrm{~mm}$, and $\mathrm{z}=-4.86 \pm 1.25 \mathrm{~mm}$ for the STN; and $\mathrm{x}=$ $20.33 \pm 1.36 \mathrm{~mm}, \mathrm{y}=-3.4 \pm 1.6 \mathrm{~mm}$, and $\mathrm{z}=-4.04 \pm 1.15$ $\mathrm{mm}$ for the GPi.

After the DBS stimulator was turned on, a movement disorders neurologist (P.H.) periodically adjusted the internal pulse generator parameters to empirically achieve optimized PD symptom control and the absence of marked side effects; she also made appropriate changes in the PD pharmacological therapy. The mean amplitude of DBS was $3.35 \mathrm{~V}$ (range 2.2-4.4 V), with $70 \%$ of the patients having a $90-\mu \mathrm{m}$ pulse width $(5$ patients at $60 \mu \mathrm{m}$, 1 at $120 \mu \mathrm{m}$, and 2 at $150 \mu \mathrm{m})$ and $77 \%$ of the patients having a rate of $185 \mathrm{~Hz}$ (the rest of the patients were divided between 130 and $150 \mathrm{~Hz}$ ).

\section{Experimental Conditions and Procedures}

Before surgery, patients in the PD-DBS group were tested in 2 conditions in the following order: 1) off condition: the practical off antiparkinson medication state with a medication washout of at least 12 hours; and 2) DOPA condition: on levodopa medication, with testing at least an hour after taking the usual dose. Patients were tested again 6 months after surgery in 4 different conditions in the following order: 1) DBS condition: testing with DBS stimulators turned on after a 12-hour levodopa washout; 2) off condition: testing with DBS stimulators turned off at least 30 minutes before testing and off medication; 3) DOPA condition: testing 1 hour after taking the usual dose of levodopa with stimulators off; and 4) DBS+DOPA condition: testing at least 30 minutes after DBS stimulators were switched on again while still on medication.

At the start of each trial, patients stood with each foot on separate side-by-side force plates. They were instruct- 


\section{Effect of deep brain stimulation on step initiation}

TABLE 1: Summary of personal and clinical data on patients with PD*

\begin{tabular}{|c|c|c|c|c|c|c|c|}
\hline \multirow{2}{*}{$\begin{array}{l}\text { Target } \\
\text { Site }\end{array}$} & \multirow{2}{*}{$\begin{array}{c}\text { No. of } \\
\text { Patients }\end{array}$} & \multirow[b]{2}{*}{ Sex Distribution } & \multirow{2}{*}{$\begin{array}{c}\text { Disease Duration } \\
\text { (yrs) }\end{array}$} & \multirow[b]{2}{*}{ Age (yrs) } & \multirow{2}{*}{$\begin{array}{l}\text { Hoehn \& Yahr } \\
\text { Score } †\end{array}$} & \multicolumn{2}{|c|}{ Levodopa-Equivalent Dose (mg/day) } \\
\hline & & & & & & Presurgery & Postsurgery \\
\hline GPi & 14 & $13 \mathrm{M}, 1 \mathrm{~F}$ & $12.9 \pm 10.17$ & $61.1 \pm 8.4$ & $\begin{array}{l}\text { Off } 3.5 \pm 0.9, \text { On } \\
\quad 2.9 \pm 0.7\end{array}$ & $1305.9 \pm 667.4$ & $1097.3 \pm 361.7$ \\
\hline STN & 15 & $11 \mathrm{M}, 4 \mathrm{~F}$ & $11.9 \pm 4.8$ & $61.4 \pm 5.5$ & $\begin{array}{l}\text { Off } 3.2 \pm 0.7, \text { On } \\
2.3 \pm 0.6\end{array}$ & $1313.1 \pm 670.2$ & $950.6 \pm 512.1 \neq$ \\
\hline
\end{tabular}

ed to voluntarily take 2 steps forward, starting with the right foot. Steps were self-initiated at the patient's normal, comfortable pace. Three trials of step initiation were performed, starting with the feet parallel, approximately $25 \mathrm{~cm}$ apart. Initial stance was made consistent across trials by having patients stand within tracings of their foot outlines on the force plates and by coaching them to maintain their habitual anteroposterior and mediolateral COP position as monitored by the experimenters on an oscilloscope.

To check for any practice or fatigue effects due to repeated trials, the CTRL group completed 1 test session with 4 sets of trials to match the 4 postsurgery conditions of the patients in the PD-DBS group. The PD-C group was tested (on and off medication) at baseline and 6 months later to determine if changes in step initiation were related to disease progression.

Clinical evaluation using the motor section (III) of the UPDRS ${ }^{14}$ and the Hoehn and Yahr scale ${ }^{21}$ was performed immediately before each test condition.

\section{Data Acquisition and Analysis}

The APA phase of step initiation was measured by the lateral COP excursion toward the initial swing limb. Magnitude of the APA was measured from the baseline to the peak lateral COP (peak COP). The APA duration was measured as the time from the onset of the first measurable change in lateral COP (APA start) to the time of foot-off (that is, the instant the vertical forces detected that the initial swing limb left the force plate). Four vertical force sensors under each force plate were used to calculate the position of the total body COP. Data from the force platform were acquired at $480 \mathrm{~Hz}$ and were lowpass filtered at $50 \mathrm{~Hz}$.

The step initiation phase was characterized by the length and velocity of the first step measured via reflective markers placed on the right lateral malleolus that were detected by infrared cameras (Motion Analysis, Inc.). Velocity was computed as the ratio between step length and step duration. Kinematic data were acquired at $60 \mathrm{~Hz}$.

The median of 3 step initiation trials was used to represent performance in each condition. For details on data acquisition and APA parameters, see Rocchi et al. ${ }^{36}$

\section{Statistical Analyses}

To calculate the statistical significance of comparisons made from our data set, a repeated-measures ANOVA was used after checking for normal distribution of the data. Tests were performed on all of the APAs and steps.

For comparisons between the PD-DBS and CTRL groups, we applied a repeated-measures ANOVA using the group as the between factor.

A preliminary evaluation was performed to detect possible differences between patients with DBS in the STN or the GPi. To this aim, DBS site was the between factor and treatment condition was the within factor.

To detect differences due to the surgical procedure (pre- and postsurgery conditions) and the 4 different treatment conditions, an ANOVA was performed separately on the STN and GPi groups, considering only within factor(s) to evaluate: 1) presurgery responsiveness to levodopa (treatment as the within factor considering only presurgery data); 2) postsurgery responsiveness to levodopa, to DBS, and to their interaction (treatment as the within factor considering only postsurgery data); and 3) pre- and postsurgery comparisons, including the off states and the best-treatment states, namely, DOPA presurgery and DBS+DOPA postsurgery (surgery and treatment as within factors). In points 2 and 3 , a post hoc multiple comparison test (all pairwise by the Fisher least significant difference test) was performed to detect pairs of means with statistically significant differences.

The repeated-measures ANOVA was also used to assess changes between baseline and 6 months postsurgery in the PD-C group and over repeated sessions within a day in the CTRL group. All statistical analyses were made using NCSS software.

\section{Results}

\section{Anticipatory Postural Adjustment Phase}

In the CTRL group (28 healthy control volunteers), APA magnitude and duration were not significantly different across the 4 sequential sessions. For this reason, the CRTL group mean ( \pm standard error of the mean) for the first of the 4 experimental sessions was used in all figures.

In both presurgery conditions and all 4 postsurgery conditions, APA magnitude was smaller and APA dura- 
tion was longer in the PD-DBS group than in the CTRL group ( $\mathrm{p}<0.01$ ). Representative APAs (lateral COP excursion) during step initiation are illustrated in Fig. 1A for a healthy control volunteer and a PD-DBS patient.

The STN and GPi groups did not differ in response of APA variables across conditions, although differences were found between treatment conditions, as detailed below and shown in Fig. 1B and C.

Anticipatory Postural Adjustment Magnitude. Before surgery, the peak COP was significantly increased by levodopa $(\mathrm{p}<0.01)$ in both the STN and GPi groups, although it was significantly smaller in PD-DBS patients

A

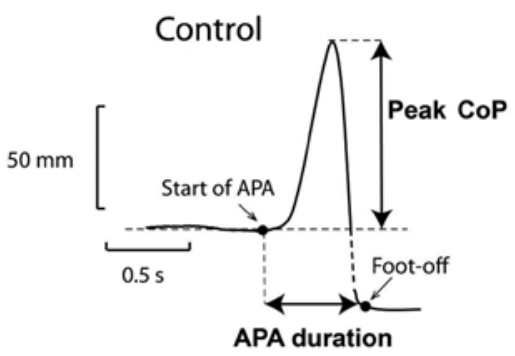

B

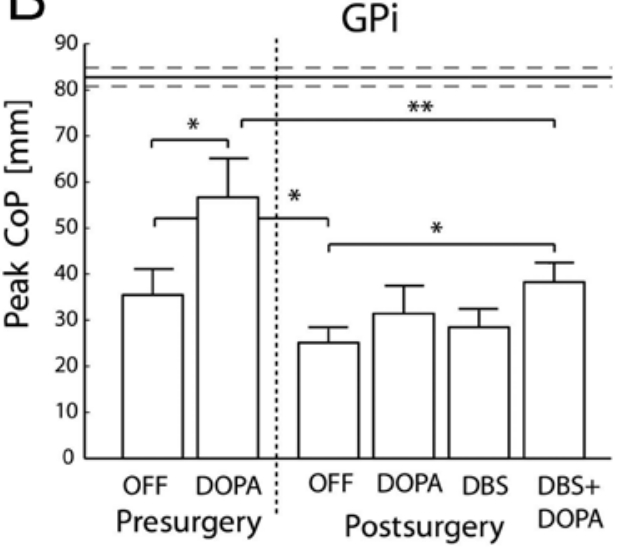

C

GPi

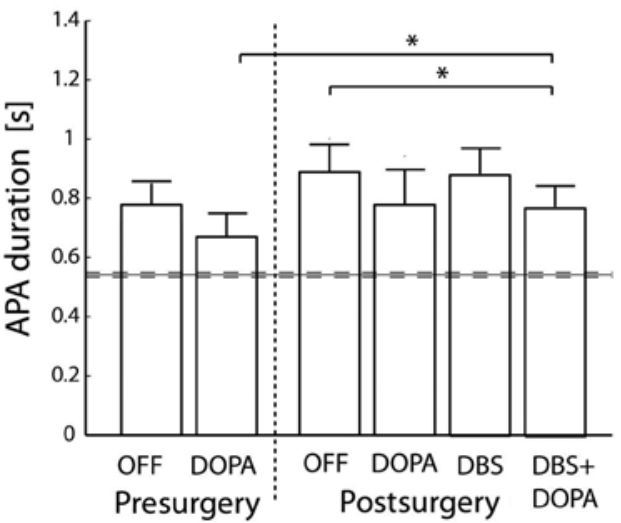

than in the CTRL group. These results were consistent with our findings in a previous study. ${ }^{36}$ After surgery, the DOPA, DBS, and DBS+DOPA conditions had larger peak COP values than those for the postsurgery off condition for both the STN and GPi groups, but only the DBS+DOPA condition reached significance ( $p<0.05$ for both groups; Fig. 1B). Peak COP in the postsurgery off condition was less than that in the presurgery off condition. Comparison of the best-treatment states before (DOPA) and after (DBS+DOPA) surgery showed that peak COP in the postsurgery condition was significantly less than that in the presurgery condition for both STN

\section{PD Patient}
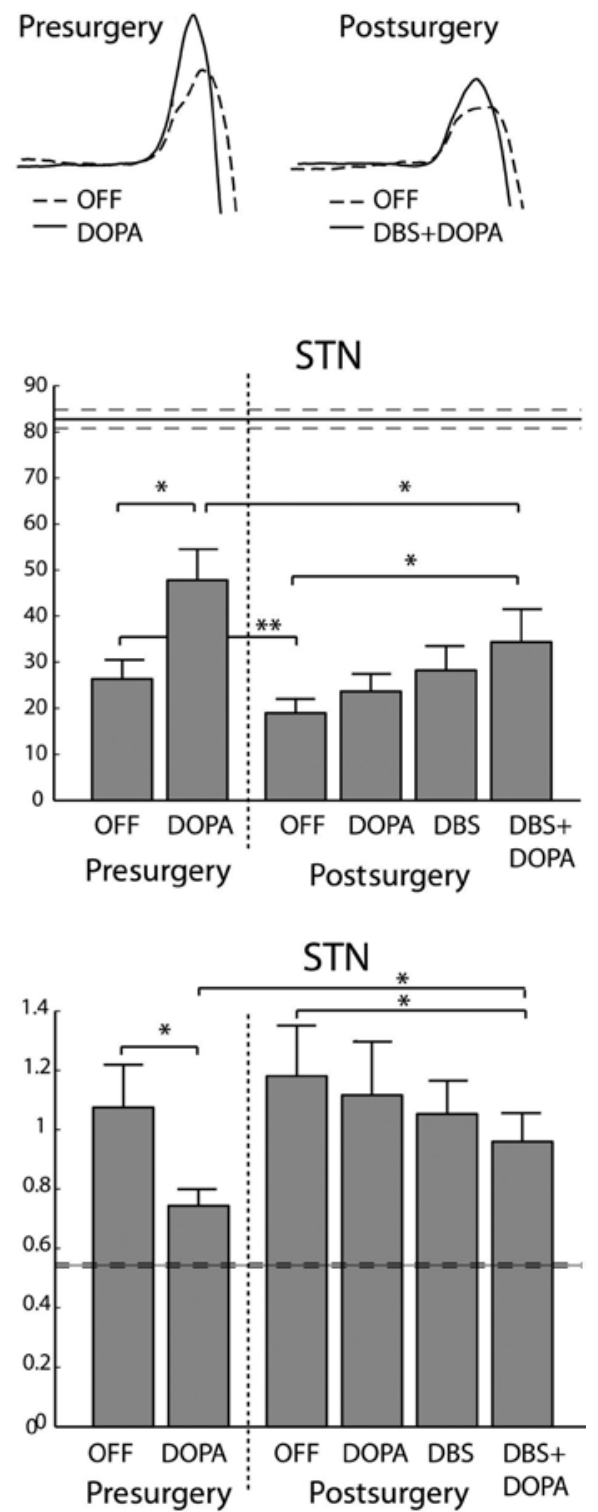

FIG. 1. A: Representative time series of lateral COP excursion during the APA. B: Bar graph demonstrating peak COP, that is, peak of lateral COP excursion during APA. C: Bar graph showing APA duration, that is, the time necessary to take the first step from APA onset. Data are presented as the means \pm standard errors of the means. Horizontal lines in $B$ and $C$ represent values for healthy control volunteers (CTRL). ${ }^{*} p<0.05 ;{ }^{* *} p<0.005$. OFF postsurgery = DBS off and levodopa off; OFF presurgery = levodopa off. 
and GPi groups. In fact, after surgery, peak COP was no longer increased by levodopa in either the STN or GPi group.

Anticipatory Postural Adjustment Duration. Before surgery, levodopa decreased the APA duration closer to normal values but only in the STN group $(\mathrm{p}<0.5)$. While this is consistent with levodopa effects in previous studies, ${ }^{36}$ the GPi group did not have a significant levodopa effect, perhaps because APA duration in the presurgery off condition was already close to the CTRL group level. The best-treatment condition postsurgery (DBS+DOPA) improved (shortened) APA duration compared with the postsurgery off condition ( $p<0.05$ in both STN and GPi groups). The APA duration in the postsurgery off condition was longer than that in the presurgery off condition but did not reach statistical significance. Anticipatory postural adjustment duration was better (shorter) in the presurgery DOPA condition than in the DBS+DOPA condition (best-treatment states, $\mathrm{p}<0.05$ for both the STN and GPi groups; Fig. 1C).

To better illustrate changes related to the surgery effect (presurgery off condition vs postsurgery off condition) and the best-treatment effect (presurgery DOPA vs postsurgery DBS+DOPA), we plotted the individual patient values along with group means (Fig. 2). Note that the peak APA magnitude reduction after surgery in the off condition for both the STN and GPi groups was statistically significant $(\mathrm{p}<0.05$ in GPi and $\mathrm{p}<0.005$ in STN, post hoc multiple comparison test), and this reduction was consistent for all but 3 patients in the GPi group and all but 2 patients in the STN group. Similarly, a comparison between best-treatment conditions showed that DBS+DOPA was not as effective as levodopa presurgery in improving APA size $(\mathrm{p}<0.005$ in GPi and $\mathrm{p}<0.05$ in STN, post hoc multiple comparison test). Worsening of the APA amplitude after surgery was true for all but 2 patients in the GPi group and all but 3 patients in the STN group.

The peak COP and APA durations in the PD-C group were similar to those in the PD-DBS group before surgery, both when evaluated at baseline and at the 6-month follow-up (Fig. 3). In both of these groups, the peak COP magnitude increased and APA duration decreased in the DOPA condition compared with those in the off condition $(\mathrm{p}<0.05)$. No differences in these 2 measures were found between baseline and 6-month follow-up testing.

\section{Stepping Phase}

The repeated-measures ANOVA did not detect differences between the STN and GPi groups in terms of the surgery effect or response to treatments for both kinematic variables, that is, step length and velocity (Fig. 4). Parkinson disease reduced the length $(\mathrm{p}<0.01)$ and velocity $(\mathrm{p}<0.01)$ of the first step compared with healthy controls. Before surgery, levodopa improved the stepping phase, since both length and velocity of the first step increased as compared with those in the off condition (length: $\mathrm{p}<$ 0.05 for STN and GPi groups; velocity: $\mathrm{p}<0.05$ for GPi group and $p<0.005$ for STN group). For both the STN and GPi groups, length and velocity in the DBS+DOPA condition increased compared with those in the postsur-
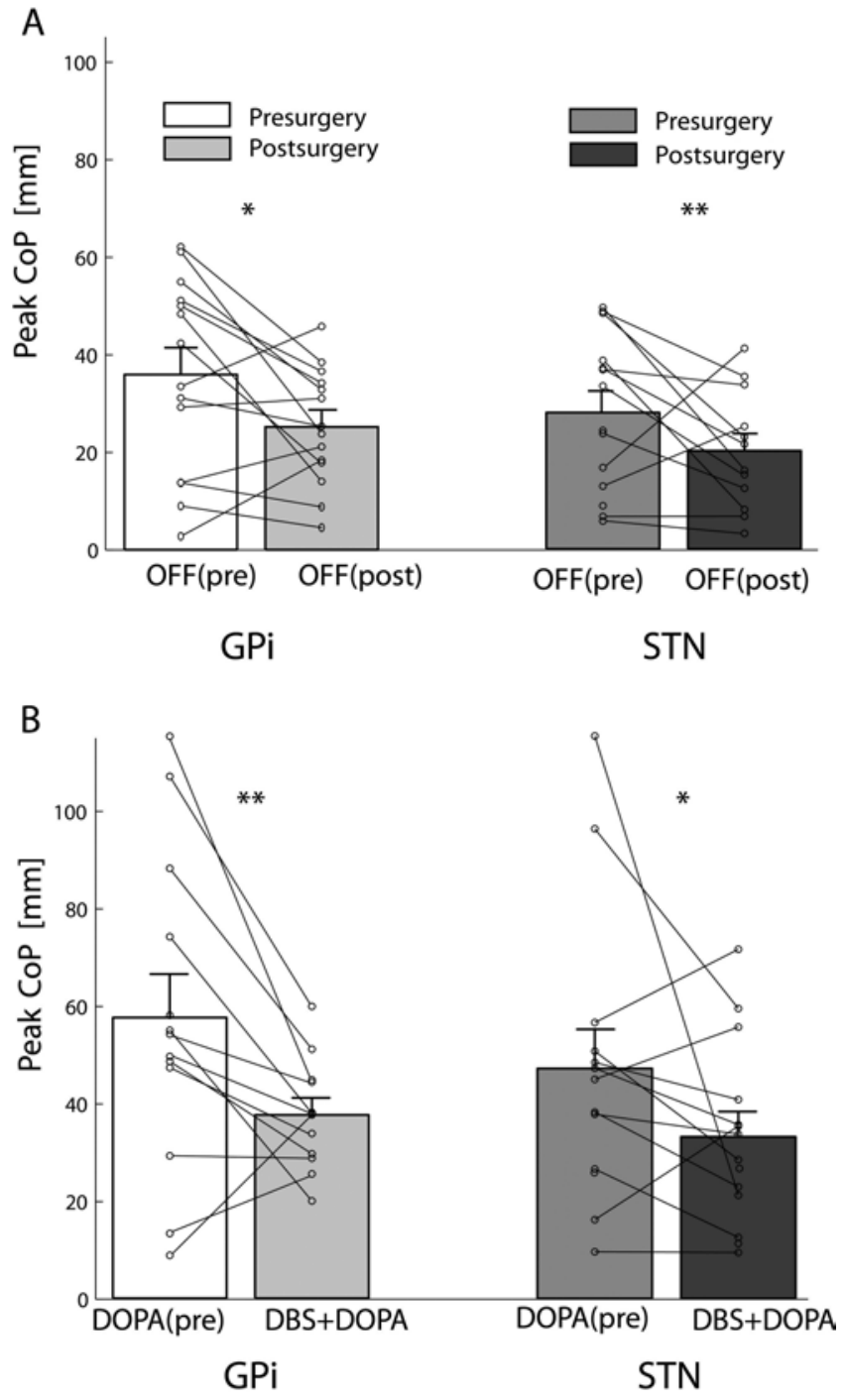

FIG. 2. Anticipatory postural adjustment amplitudes postsurgery compared with presurgery. A: Graph demonstrating off states to investigate surgery effect. B: Graph showing DOPA condition presurgery [DOPA(pre)] and DBS+DOPA posturgery to compare the best medical management states. Values are expressed as the means \pm standard errors of the means, and individual patient values are plotted. ${ }^{*} p<0.05 ;{ }^{* *} p<0.005$. OFF(post) = postsurgery off condition (DBS off and levodopa off); OFF(pre) = presurgery off condition (levodopa off).

gery off condition $(\mathrm{p}<0.05)$. For the GPi group, length and velocity in the DBS+DOPA condition were restored to levels similar to those in the presurgery DOPA condition. For the STN group, although step length in the DBS+DOPA condition was restored to the presurgery DOPA level, velocity remained lower than in the presurgery DOPA condition $(\mathrm{p}<0.05)$.

\section{Clinical Evaluation}

Before surgery, clinical signs, as measured using the motor section of the UPDRS, were greatly ameliorated in the DOPA condition compared with the off condition $(p<0.001)$. After surgery, results confirmed a reduction in clinical signs on any of the therapies compared with 
A

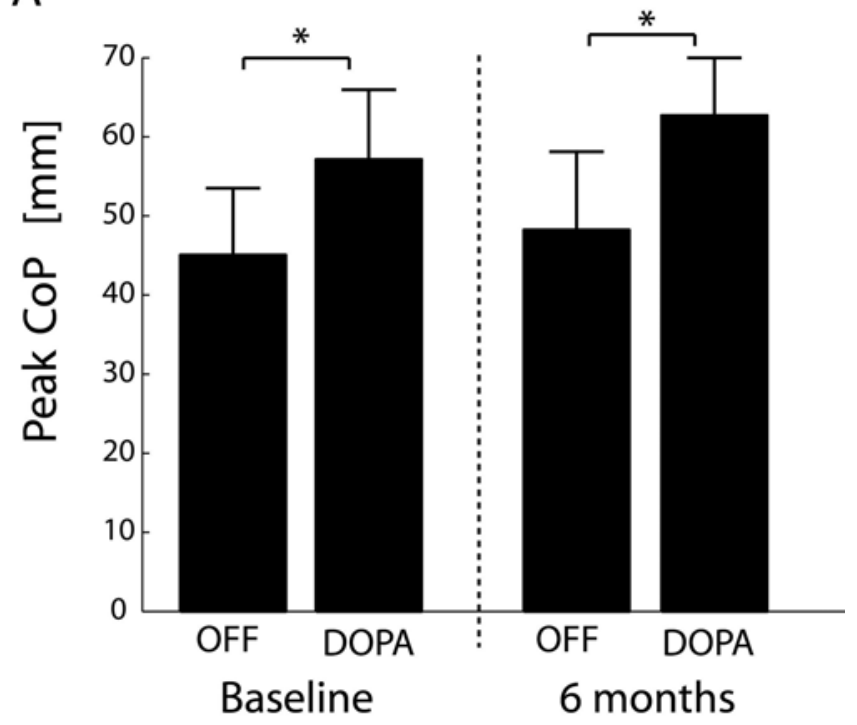

B

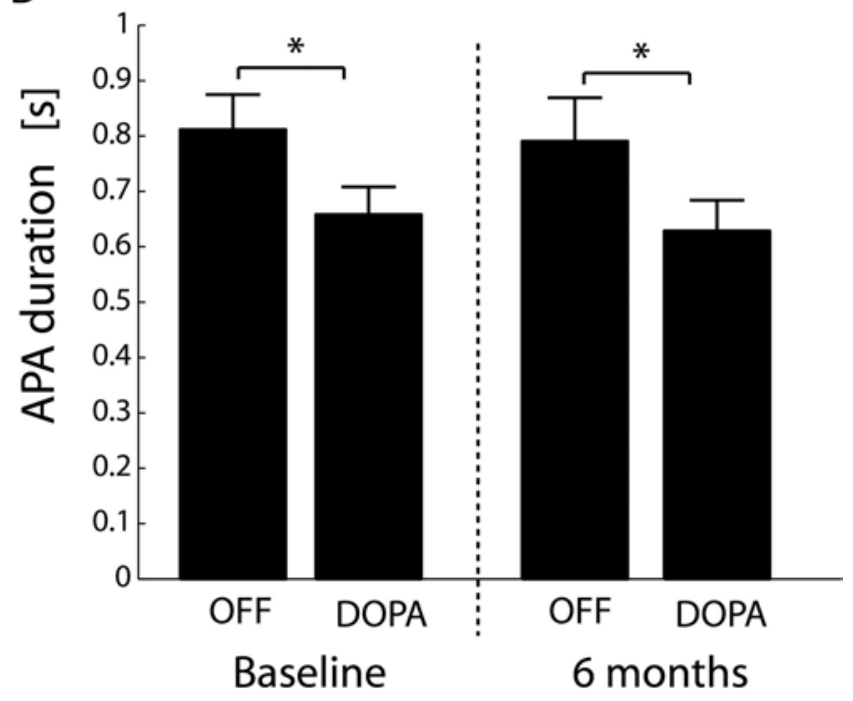

Fıg. 3. Bar graphs showing APA amplitude (A) and duration (B) in the PD-C group (control volunteers with PD who met the criteria for DBS surgery), evaluated at baseline and 6 months later. ${ }^{*} p<0.05$. DOPA = levodopa on; OFF = levodopa off.

the off condition $(\mathrm{p}<0.001)$ for both the STN and GPi groups. In fact, the administration of levodopa at the patient's usual dose along with activation of the stimulator resulted in a UPDRS motor score comparable to that in the presurgery DOPA condition. Values and more detailed data on the UPDRS motor scores in the 6 tested conditions are presented in Table 2.

The PD-C patients had a UPDRS motor score comparable to that in the PD-DBS group, with $45.5 \pm 16$ and $22.9 \pm 8.9$ at baseline and $45.9 \pm 12.8$ and $24.2 \pm 9.6$ after 6 months (off and on levodopa, respectively). The levodopa-equivalent dose in the $\mathrm{PD}-\mathrm{C}$ group was 1234 $\pm 450 \mathrm{mg} /$ day at baseline and $1144 \pm 326 \mathrm{mg} /$ day after 6 months, values comparable to those in PD-DBS patients before surgery (Table 1).

\section{Discussion}

Our results showed that 6 months of DBS can impair step initiation. Prior to surgery, the PD-DBS patients showed smaller than normal lateral weight shifts associated with APAs in preparation for a step as well as smaller and slower steps, which is consistent with findings in the literature. ${ }^{7,36}$ Also consistent with the literature, APA amplitude, step size, and velocity were improved with levodopa before surgery. ${ }^{5,36,37}$ However, 6 months after DBS surgery, the size of the APA was smaller, the duration of step initiation was longer, and the presurgery improvement with levodopa was not restored.

The APAs were similarly impaired by DBS surgery in both the GPi and STN groups. In contrast, automatic postural responses to external perturbations in the same patients were impaired by DBS surgery in the STN group but not in the GPi group. ${ }^{40}$ Similarly, jaw movements were impaired by DBS surgery only in the STN group but not in the GPi group. ${ }^{35}$ Thus, DBS in the STN impaired APAs, automatic postural responses, and jaw movements, whereas DBS in the GPi impaired APAs but did not change automatic postural responses or jaw movements. These results suggest that the STN plays a similar role for these axial motor behaviors, whereas GPi circuitry may play different roles.

Two previous studies have focused on the effects of DBS in the STN on step initiation. ${ }^{28,38}$ Authors of both of these studies concluded that DBS in the STN improved step initiation. However, it is important to note that only postsurgery data were considered in these studies. We found similar small improvements in APAs in the DBS+DOPA condition, as compared with those in the postsurgery off condition (Fig. 1). Nevertheless, APA amplitudes and durations were worse in the DBS+DOPA condition than in the presurgical DOPA condition. To our knowledge, our study is the first to compare movement initiation both pre- and post-DBS surgery.

Surprisingly, we found that levodopa and DBS produced different effects on step initiation. In fact, whereas levodopa improved APAs for step initiation, DBS in either the STN or the GPi impaired them. Previous studies showing that the effects of DBS on motor tasks are similar to those of levodopa are consistent with a simple circuitry model of PD.,12 This model suggests that DBS and dopamine both inhibit excessive tonic basal ganglia outflow to thalamocortical and brainstem circuits. In contrast, in our study the positive effects of levodopa and the negative effects of DBS on APAs for step initiation suggest that DBS may have some nondopaminergic effects. In fact, the poor responsiveness of APAs to levodopa after surgery, as compared with before surgery, suggests that the DBS surgery itself impairs the action of levodopa on APAs.

A poor APA response to levodopa after surgery is probably not caused by a reduction in levodopa. In our patients, the levodopa-equivalent dose was slightly decreased after surgery as compared with before, with a significant change $(p=0.02)$ only in the STN group, which is consistent with findings in the literature. ? However, a correlation between the decline in the APA peak and a change in the levodopa dose before and after sur- 

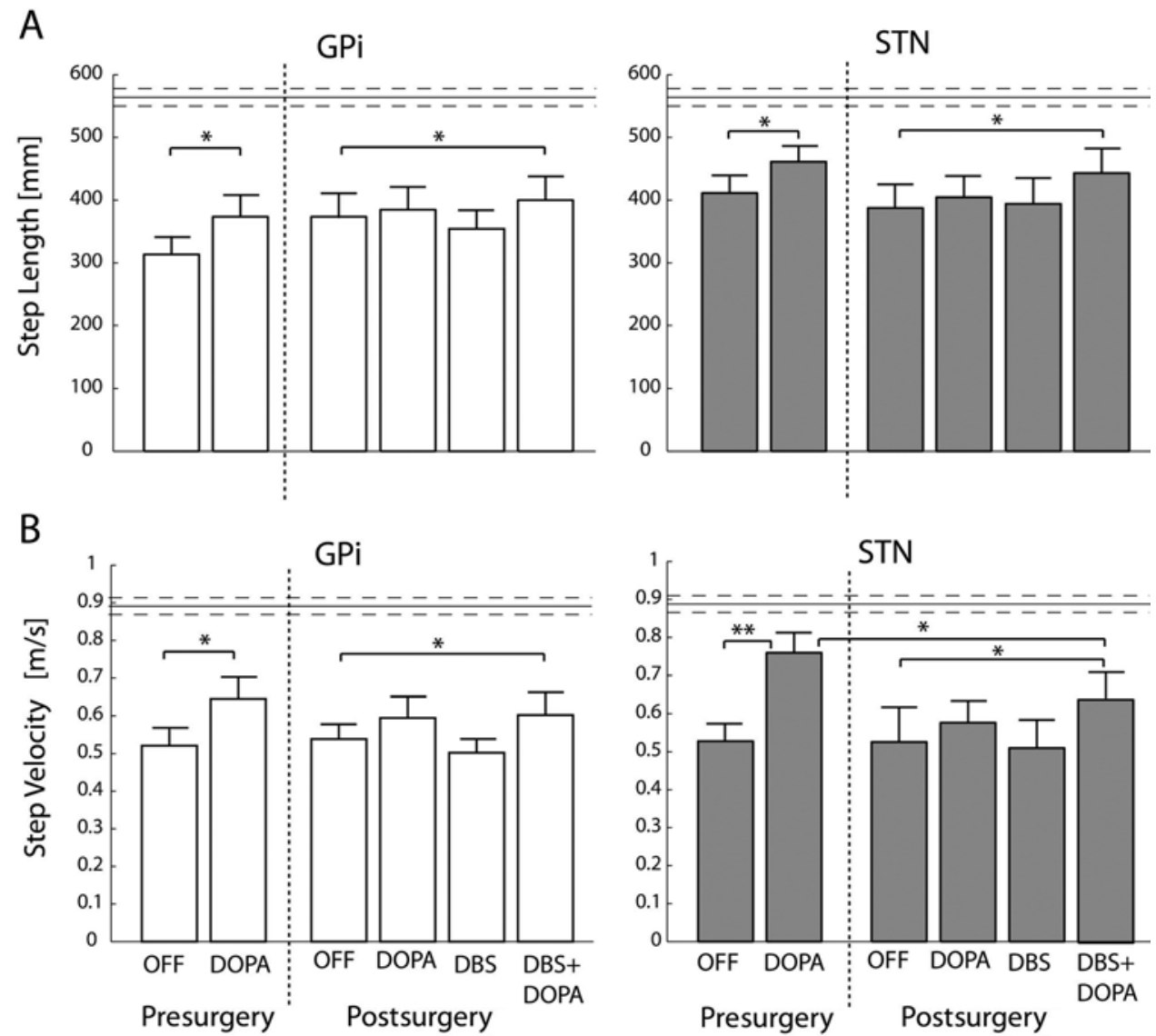

FIG. 4. Bar graphs showing kinematics of the first step: step length (A) and step velocity (B). Data are expressed as the means \pm standard errors of the means. Horizontal lines represent values for healthy control volunteers (CTRL). ${ }^{*} p<0.05 ;{ }^{* *} p<0.005$. OFF postsurgery = DBS off and levodopa off; OFF presurgery = levodopa off.

gery was not significant $(p=0.2$ in GPi group, $p=0.75$ in STN group). Although we cannot know the reason for the reduced levodopa effect after surgery, it is possible that the stabilization of abnormal basal ganglia network oscillations with DBS may in some way limit the effects of levodopa on specific postural tasks, such as step initiation, that depend on the interaction of the pedunculopontine nucleus and other locomotor brainstem areas and the supplementary motor area..$^{32}$ Although the physiological mechanisms responsible for DBS effects are unknown, long-term plastic changes of synapses and circuitry have been observed as a result of chronic DBS., 427

We do not know whether the impairment in step preparation after DBS surgery was related to a lesion effect or to 6 months of continuous high-frequency stimula- tion in the basal ganglia, resulting in long-lasting alterations of circuits that are important for postural preparation, even when the stimulators were turned off. Future studies should compare step initiation before and after surgery but before the stimulators have been turned on to differentiate between a surgical procedure effect versus a chronic stimulation effect.

Our comparison with a PD control group that did not have DBS surgery dispels the likelihood that the worsening step initiation after DBS surgery is attributable to disease progression across 6 months. In fact, the PD controls had better step initiation with levodopa at both baseline and 6 months later, unlike the patients who underwent DBS surgery, who only improved with levodopa before surgery.

TABLE 2: The UPDRS motor score pre- and postsurgery*

\begin{tabular}{|c|c|c|c|c|c|c|}
\hline \multirow[b]{2}{*}{ DBS Site } & \multicolumn{2}{|c|}{ Presurgery† } & \multicolumn{4}{|c|}{ Postsurgery } \\
\hline & Off & DOPA & Off & DBS & DOPA & DBS+DOPA \\
\hline GPi & $50.9 \pm 18.2$ & $28.5 \pm 14.4$ & $47.1 \pm 17.0$ & $34.5 \pm 18.4$ & $31.6 \pm 12.8$ & $22.8 \pm 12.7$ \\
\hline STN & $49.0 \pm 11.9$ & $21.2 \pm 11.0$ & $51.1 \pm 20.9$ & $33.5 \pm 11.5$ & $35.2 \pm 15.1$ & $20.6 \pm 8.4$ \\
\hline
\end{tabular}

* Values are expressed as the means \pm standard deviations.

$\dagger p<0.001$, off vs DOPA.

$\ddagger p<0.01$, off vs DOPA or DBS; $p<0.001$, off vs DBS+DOPA; $p<0.01$, DBS or DOPA vs DBS+DOPA. 
Interestingly, movement execution was not as disrupted by DBS as postural preparation prior to movement. Specifically, step length was the same in the besttreatment states before and after surgery in both the GPi and STN groups, and step velocity was the same before and after surgery in the GPi group. The different effects of DBS on postural preparation and stepping suggest independent motor pathways for the preparation and execution of movement. ${ }^{13}$ Studies recording neural activity in the reticular formation of the feline brainstem showed different neurons active during APAs and the movement phase. ${ }^{13}$ In addition, recent studies demonstrated that APAs may be triggered independently of the step by an acoustic startle. ${ }^{30,39}$ Moreover, lesions isolated to the supplementary motor area resulted in a loss of APAs prior to arm movements, which were not affected by the lesion. ${ }^{18,31}$

From a clinical viewpoint, the motor score of the UPDRS was the same before and after surgery in the best-treatment conditions (DOPA presurgery and DBS+DOPA postsurgery), which is consistent with findings in the literature. ${ }^{16}$ The UPDRS does not appear to be a sensitive measure of APAs prior to step initiation, so additional clinical tests of balance should be considered. ${ }^{17}$ The lack of quantitative improvement in postural preparation for a step after DBS surgery is consistent with clinical studies suggesting that DBS may not be as effective a treatment for axial signs in patients with PD as it is for tremor, rigidity, and bradykinesia. ${ }^{3,5,24}$ In fact, the Veterans Affairs/National Institute of Neurological Disorders and Stroke multicenter clinical trial comparing the STN and GPi as DBS sites also showed an increase in injurious falls after DBS surgery, especially for the STN group, although both groups otherwise benefited from surgery with fewer on/off fluctuations. . $^{12,44}$

One limitation of our study was the difference in baseline APA duration and magnitude between the STN and GPi groups. This bias did not correlate with diversity in clinical signs and appeared even though PDDBS patients were randomized to STN and GPi surgery sites. Although the sample in our study is quite large, when compared with populations in previous analogous works, ${ }^{15,20,23,28,29,38}$ our limited number of participants represents a limitation. Regardless, our study does have the advantage of considering both pre- and postsurgery conditions as well as both STN and GPi sites. In contrast, previous DBS studies examining step initiation and gait have only included postsurgery conditions for one DBS site. It is also noteworthy that we evaluated performance in several different axial tasks, such as speech ${ }^{34}$ and postural responses,$^{40}$ as well as APAs prior to step initiation. A comprehensive evaluation of PD-DBS patients using a variety of motor tasks will allow a better understanding of the effects of DBS and the role of the basal ganglia in axial motor control.

\section{Conclusions}

Our study highlights the importance of comparing motor behavior pre- and postsurgery to determine the effects of DBS on patients with PD. Despite the many benefits of DBS for patients with PD, our study indicates potential postural issues associated with the initiation of walking, which may worsen start hesitation or freezing after surgery. ${ }^{33}$

\section{Disclosure}

This research was supported by National Institute on Aging Grant Nos. AG19706 and AG006457.

Author contributions to the study and manuscript preparation include the following. Conception and design: Hogarth, Horak. Acquisition of data: Rocchi, Carlson-Kuhta, Burchiel, Hogarth, Horak. Analysis and interpretation of data: Rocchi, Horak. Drafting the article: Rocchi, Carlson-Kuhta, Horak. Critically revising the article: all authors. Reviewed submitted version of manuscript: all authors. Approved the final version of the manuscript on behalf of all authors: Rocchi. Study supervision: Horak.

\section{Acknowledgments}

The authors acknowledge the assistance of Triana Nagel for patient scheduling and data collection, Marilee Stephens and Lesley Silar for help with data collection, and Dr. Ali Samii for referring patients from the Seattle location. Magnetic resonance images to verify electrode placement were available courtesy of the VA CSP Study \#468, A Comparison of Best Medical Therapy and Deep Brain Stimulation of Subthalamic Nucleus and Globus Pallidus for the Treatment of Parkinson's Disease.

\section{References}

1. Albin RL, Young AB, Penney JB: The functional anatomy of disorders of the basal ganglia. Trends Neurosci 18:63-64, 1995

2. Anderson VC, Burchiel KJ, Hogarth P, Favre J, Hammerstad JP: Pallidal vs subthalamic nucleus deep brain stimulation in Parkinson disease. Arch Neurol 62:554-560, 2005

3. Bejjani BP, Gervais D, Arnulf I, Papadopoulos S, Demeret S, Bonnet AM, et al: Axial parkinsonian symptoms can be improved: the role of levodopa and bilateral subthalamic stimulation. J Neurol Neurosurg Psychiatry 68:595-600, 2000

4. Beuter A, Modolo J: Delayed and lasting effects of deep brain stimulation on locomotion in Parkinson's disease. Chaos 19: 026114, 2009

5. Bloem BR, Hausdorff JM, Visser JE, Giladi N: Falls and freezing of gait in Parkinson's disease: a review of two interconnected, episodic phenomena. Mov Disord 19:871-884, 2004

6. Bouisset S, Richardson J, Zattara M: Do anticipatory postural adjustments occurring in different segments of the postural chain follow the same organisational rule for different task movement velocities, independently of the inertial load value? Exp Brain Res 132:79-86, 2000

7. Bronstein JM, Tagliati M, Alterman RL, Lozano AM, Volkmann J, Stefani A, et al: Deep brain stimulation for Parkinson disease: an expert consensus and review of key issues. Arch Neurol 68:165-171, 2011

8. Burchiel KJ, Anderson VC, Favre J, Hammerstad JP: Comparison of pallidal and subthalamic nucleus deep brain stimulation for advanced Parkinson's disease: results of a randomized, blinded pilot study. Neurosurgery 45:1375-1384, 1999

9. Burleigh-Jacobs A, Horak FB, Nutt JG, Obeso JA: Step initiation in Parkinson's disease: influence of levodopa and external sensory triggers. Mov Disord 12:206-215, 1997

10. Couillandre A, Brenière Y, Maton B: Is human gait initiation program affected by a reduction of the postural basis? Neurosci Lett 285:150-154, 2000

11. Crenna P, Carpinella I, Rabuffetti M, Rizzone M, Lopiano L, Lanotte M, et al: Impact of subthalamic nucleus stimulation 
on the initiation of gait in Parkinson's disease. Exp Brain Res 172:519-532, 2006

12. DeLong MR: Primate models of movement disorders of basal ganglia origin. Trends Neurosci 13:281-285, 1990

13. Drew T, Prentice S, Schepens B: Cortical and brainstem control of locomotion. Prog Brain Res 143:251-261, 2004

14. Fahn S, Elton RL: Unified Parkinson's Disease Rating Scale, in Fahn S, Marsden CD, Calne DB, et al (eds): Recent Developments in Parkinson's Disease. Florham Park, NJ: Macmillan, 1987, Vol 2, pp 153-163

15. Ferrarin M, Rizzone M, Bergamasco B, Lanotte M, Recalcati M, Pedotti A, et al: Effects of bilateral subthalamic stimulation on gait kinematics and kinetics in Parkinson's disease. Exp Brain Res 160:517-527, 2005

16. Follett KA, Weaver FM, Stern M, Hur K, Harris CL, Luo P, et al: Pallidal versus subthalamic deep-brain stimulation for Parkinson's disease. N Engl J Med 362:2077-2091, 2010

17. Franchignoni F, Horak F, Godi M, Nardone A, Giordano A: Using psychometric techniques to improve the Balance Evaluation Systems Test: the mini-BESTest. J Rehabil Med 42: 323-331, 2010

18. Gurfinkel' VS, El'ner AM: [Participation of the secondary motor area of the frontal lobe of the brain in organizing postural components of human voluntary movement.] Neirofiziologiia 20:7-15, 1988 (Russian)

19. Halliday SE, Winter DA, Frank JS, Patla AE, Prince F: The initiation of gait in young, elderly, and Parkinson's disease subjects. Gait Posture 8:8-14, 1998

20. Hausdorff JM, Gruendlinger L, Scollins L, O'Herron S, Tarsy D: Deep brain stimulation effects on gait variability in Parkinson's disease. Mov Disord 24:1688-1692, 2009

21. Hoehn MM, Yahr MD: Parkinsonism: onset, progression and mortality. Neurology 17:427-442, 1967

22. Jacobs JV, Lou JS, Kraakevik JA, Horak FB: The supplementary motor area contributes to the timing of the anticipatory postural adjustment during step initiation in participants with and without Parkinson's disease. Neuroscience 164:877-885, 2009

23. Johnsen EL, Mogensen PH, Sunde NA, Østergaard K: Improved asymmetry of gait in Parkinson's disease with DBS: gait and postural instability in Parkinson's disease treated with bilateral deep brain stimulation in the subthalamic nucleus. Mov Disord 24:590-597, 2009

24. Kleiner-Fisman G, Fisman DN, Sime E, Saint-Cyr JA, Lozano AM, Lang AE: Long-term follow up of bilateral deep brain stimulation of the subthalamic nucleus in patients with advanced Parkinson disease. J Neurosurg 99:489-495, 2003

25. Krack P, Batir A, Van Blercom N, Chabardes S, Fraix V, Ardouin $\mathrm{C}$, et al: Five-year follow-up of bilateral stimulation of the subthalamic nucleus in advanced Parkinson's disease. $\mathbf{N}$ Engl J Med 349:1925-1934, 2003

26. Lepers R, Brenière Y: The role of anticipatory postural adjustments and gravity in gait initiation. Exp Brain Res 107:118124,1995

27. Li XH, Wang JY, Gao G, Chang JY, Woodward DJ, Luo F: High-frequency stimulation of the subthalamic nucleus restores neural and behavioral functions during reaction time task in a rat model of Parkinson's disease. J Neurosci Res 88: 1510-1521, 2010

28. Liu W, McIntire K, Kim SH, Zhang J, Dascalos S, Lyons KE, et al: Bilateral subthalamic stimulation improves gait initiation in patients with Parkinson's disease. Gait Posture 23: 492-498, 2006

29. Lubik S, Fogel W, Tronnier V, Krause M, König J, Jost WH: Gait analysis in patients with advanced Parkinson disease: different or additive effects on gait induced by levodopa and chronic STN stimulation. J Neural Transm 113:163-173, 2006

30. MacKinnon CD, Bissig D, Chiusano J, Miller E, Rudnick L, Jager C, et al: Preparation of anticipatory postural adjustments prior to stepping. J Neurophysiol 97:4368-4379, 2007

31. Massion J: Movement, posture and equilibrium: interaction and coordination. Prog Neurobiol 38:35-56, 1992

32. Moran RJ, Mallet N, Litvak V, Dolan RJ, Magill PJ, Friston KJ, et al: Alterations in brain connectivity underlying beta oscillations in Parkinsonism. PLoS Comput Biol 7:e1002124, 2011

33. Moreau C, Defebvre L, Destée A, Bleuse S, Clement F, Blatt JL, et al: STN-DBS frequency effects on freezing of gait in advanced Parkinson disease. Neurology 71:80-84, 2008

34. Perlmutter JS, Mink JW: Deep brain stimulation. Annu Rev Neurosci 29:229-257, 2006

35. Robertson LT, St George RJ, Carlson-Kuhta P, Hogarth P, Burchiel KJ, Horak FB: Site of deep brain stimulation and jaw velocity in Parkinson disease. Clinical article. J Neurosurg 115:985-994, 2011

36. Rocchi L, Chiari L, Mancini M, Carlson-Kuhta P, Gross A, Horak FB: Step initiation in Parkinson's disease: influence of initial stance conditions. Neurosci Lett 406:128-132, 2006

37. Rochester L, Baker K, Nieuwboer A, Burn D: Targeting dopasensitive and dopa-resistant gait dysfunction in Parkinson's disease: selective responses to internal and external cues. Mov Disord 26:430-435, 2011

38. Rodriguez-Oroz MC, Obeso JA, Lang AE, Houeto JL, Pollak $\mathrm{P}$, Rehncrona $\mathrm{S}$, et al: Bilateral deep brain stimulation in Parkinson's disease: a multicentre study with 4 years follow-up. Brain 128:2240-2249, 2005

39. Rogers MW, Kennedy R, Palmer S, Pawar M, Reising M, Martinez KM, et al: Postural preparation prior to stepping in patients with Parkinson's disease. J Neurophysiol 106:915 924, 2011

40. Schepens B, Drew T: Strategies for the integration of posture and movement during reaching in the cat. J Neurophysiol 90: 3066-3086, 2003

41. Schepens B, Stapley P, Drew T: Neurons in the pontomedullary reticular formation signal posture and movement both as an integrated behavior and independently. J Neurophysiol 100:2235-2253, 2008

42. St George RJ, Carlson-Kuhta P, Burchiel KJ, Hogarth P, Frank N, Horak FB: The effects of subthalamic and pallidal deep brain stimulation on postural responses in patients with Parkinson disease. Laboratory investigation. J Neurosurg 116: 1347-1356, 2012

43. St George RJ, Nutt JG, Burchiel KJ, Horak FB: A meta-regression of the long-term effects of deep brain stimulation on balance and gait in PD. Neurology 75:1292-1299, 2010

44. Weaver FM, Follett K, Stern M, Hur K, Harris C, Marks WJ $\mathrm{Jr}$, et al: Bilateral deep brain stimulation vs best medical therapy for patients with advanced Parkinson disease: a randomized controlled trial. JAMA 301:63-73, 2009

Manuscript submitted November 9, 2011.

Accepted August 20, 2012.

Please include this information when citing this paper: published online October 5, 2012; DOI: 10.3171/2012.8.JNS112006.

Address correspondence to: Laura Rocchi, Ph.D., Department of Electronics, Computer Science \& Systems, University of Bologna, Viale del Risorgimento 2, Bologna 40136, Italy. email: 1.rocchi@ unibo.it. 